\title{
Major Issues in Adoption of Electronic Health Records
}

Myongho Yi

Department of Library and Information Science

Sangmyung University

Seoul South Korea

josephlee@smu.ac.kr

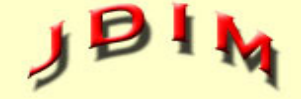

Journal of Digital Information Management
ABSTRACT: Backgrounds: The use of Electronic Health Records (EHRs) is not new, but the implementation and provision of their services are. Despite the positive aspects and promising features of EHRs use in medical and healthcare practices, there are many issues that need to be identified and addressed to expedite the adoption rate of EHRs. The objective of the study is to examine major issues which hinder EHR adoption and to present identified solutions.

Methods: Thirty-two staff members from five hospitals were surveyed in order to find common problems and obstacles/issues encountered when adopting EHRs. Members of hospital staff were also interviewed in order to pinpoint the possible problems and solutions that management staff of hospitals may encounter.

Results: The study identified possible solutions to the four major issues in EHR implementation: 1) implement ontology to ensure interoperability to share medical records among organizations, 2) provide physical, technical and managerial security controls to provide secure access for EHRs, 3) ensure business continuity of uninterrupted access to EHRs using cloud-based EHRs, 4) minimize the digital divide by providing training to users and staff.

Conclusions: Consideration of these four major issues indicated in this study will expedite hospitals and various healthcare organizations to implement and provide EHRs.
Subject Categories and Descriptors J.3 [LIFE AND MEDICAL SCIENCES]; Medical information systems

General Terms: Electronic Health Records, Medical Information Processing, Ontology

Keywords: Electronic Health Records, Interoperability, Security, Business Continuity, Digital Divide

DOI: $10.6025 / \mathrm{jdim} / 2018 / 16 / 4 / 180-191$

Received: 8 February 2018, Revised 19 March 2018, Accepted 10 March 2018

\section{Introduction}

Recent technological advancements are enabling more efficient ways to access medical information. One example of this is the use of Electronic Health Records (EHRs). EHRs are a collection of electronic health information about an individual patient (Gunter \& Terry, 2005). Electronic Health Records, Electronic Patient Records, and Electronic Medical Records are terms often used interchangeably and in relation to each other. Recording these data in electronic form is supported by laws like the Health Insurance Portability and Accountability Act (HIPAA) and The Health Information Technology for Economic and Clinical Health (HITECH) Act. HITECH was created to stimulate the adoption of EHRs in the United States. 
In the past, the traditional method of storing medical records was in paper form. However, paper form storage possesses certain limitations when it comes to accessing medical record data. Patients and hospital staff have limited access to these paper-based medical records. Some medical facilities operate under limited hours, thus restricting hours wherein data can be accessed. In the case of an emergency situation late at night, accessing medical records from medical facilities that do not run for 24 hours a day would take a lot of time and effort. Furthermore, managing medical records is not easy in paper form. Sorting and searching through medical records can be time consuming and inconvenient. Given how essential time is in the medical field, it is important that EHRs are integrated into hospital systems in order to save time. After Hurricane Katrina hit the US, the value of EHRs has been made even more relevant, since the tragedy highlighted the difficulties in accessing medical records during an emergency situation. During the disaster, medical professionals could not treat patients efficiently without easy access to their medical records (Williams \& Boren, 2008). With the use of EHRs, medical services can reach people from distant rural and urban places and those who are confined or detained in highly guarded areas i.e. jails or prisons. Thus, utilization of EHRs can lead to better share ability of medical records amongst hospitals and their medical personnel. The study conducted by Hersh (2013) showed that oncologists were able to share medical records using EHRs which will greatly assist medical treatment in such cases.

Despite the advancements made in EHRs, a lot of issues are still prevalent. In addition, the adoption rate of fully functional EHR systems is very slow in the US. Much research has been conducted to identify issues for the slow adoption rate of fully functional EHR systems as compared to partial functional EHR systems. Numerous issues have been responsible for deterring medical organizations in implementing them. However, many of these issues can be mitigated and even avoided if medical organizations addressed these issues during the implementation process. There is no comprehensive list of the main issues hindering awareness of EHR implementation. This paper presents solutions to the most pressing obstacles to EHR adoption. First, this paper identifies a comprehensive list of barriers to EHR implementation based on a comprehensive literature review. Second, this paper prioritizes four obstacles based on survey conducted in South Korea, an early adopter of EHRs. Finally, this paper presents solutions to the four obstacles. By identifying issues in EMR implementation, stakeholders and other related organizations can get a holistic view of EHRs. Hopefully, the knowledge obtained from this paper can promote the rate of their adoption. This paper discusses certain concerns and provides possible solutions to combat these issues in order to implement EHRs in the fastest and most efficient way.

\section{Background}

\subsection{Benefits of EHRs}

The four major benefits of adopting EHRs over paper-based medical records are cost, storage, security, and access (Carpathia, 2013). First, managing EHRs requires fewer staff members, and physical storage taking up large spaces for record keeping will not needed anymore. Therefore, it costs less to manage and maintain records with this method. Second, as the number of patients grow, preservation of records becomes an issue. Paper medical records need to be physically printed on papers and often take up large physical spaces to be stored. They can also easily decay and be destroyed. EHRs, on the other hand, do not need to be printed on papers that require large physical storage spaces. Third, EHRs can be stored in a more secure place such as cloud and can be monitored for any suspicious access, while paper records are mainly stored in file cabinets with less security. The files can be stolen or damaged by natural disasters such as fire and flood. To be prepared for any loss, we need to have backups. With EHRs, we can easily make copies while paper records require photocopy backups, and sorting and binding the copies together. Finally, to access paper medical records, they must be mailed or scanned and sent via email taking lots of time. However, the use of EHRs allows medical professionals to access the information they need almost instantly with the click of a button. Since time is critical in medical settings, EHR usage is more efficient when compared to paper record usage.

Currently EHRs are used within a single organization but they will be shared and utilized through a network of organizations in the near future. If this electronic medical information is shared, we can not only utilize this medical information to cure patients' diseases but also for research or statistical purposes (Chae, Yoo, \& Kim, 2010; H. Lee, 2013). With advanced network and mobile technology, we can access our EHRs and other medical services anywhere. Today's networks allow us to view high quality images of lab results and other health information, as well as give access to our medical records anytime, anywhere. Resources for transmission of EHRs are readily available (H. Lee, 2013). In addition, mobile devices are widely used to create and share sensitive health data over wireless connections.

\subsection{Electronic Health Records Adoption Rate}

Although EHRs have been recommended in the medical field, many organizations are hesitant to implement a fully functional system (Jha et al., 2009). Jha et al (2009) classify EHR adoption rate into three categories: fully functional, basic functional, and no EHRs. According to the Center for Disease Control and Prevention, the adoption rate for EHRs for office-based physician is increasing over time; however, adoption rate for fully functional EHRs is still low (Hsiao, Hing, Socey, \& Cai, 2011). The adoption rate for any EHR systems has increased from $18.2 \%$ to $41.5 \%$ but the adoption rate for fully functional systems is only $4.4 \%$ (Hsiao et al., 2011). 


\subsection{Electronic Health Records in Korea}

Many countries have switched to EHRs or have implemented various other health information technologies. Korea, known as one of the most wired countries in the world, is one of the countries where EHR has been implemented. Their network infrastructure is so advanced that various remote medical services using high-speed networks are already available. In June 2014, remote consultation and remote patient monitoring services began. Remote consultation uses telecommunications for the purpose of diagnosis or treatment of a patient at a site away from a physician. Remote patient monitoring uses devices to collect and send data to a remote diagnostic testing facility. South Korea's Cabinet passed a bill allowing doctors to utilize various health technologies to provide remote consultation and monitoring of patients. Sixty percent (60\%) of the members of the Korean Medical Association agreed with the use of these services (Lacktman, 2014). Korean medical law has permitted the saving of patients' records in electronic form since 2002. Lee, Kang, and Baik's (2009) study shows that $77.3 \%$ of the third tier hospitals have adopted EHRs as of 2009.

Many studies related to EHR adoption in Korea have been conducted. Shin and Chung (2007) presented design and implementation of an EHR based on an RFID system. Lee, Kim, and Yim (2012) addressed EHR management and preservation issues by proposing for the application of ISO 15489 standards, which covers the principles of records management, to hospital medical records management and assessment of the current quality of medical records management. Korean hospitals have used many systems such as Order Communication System (OCS) or Picture Archiving and Communication System (PACS) to manage medical records; these systems must comply with ISO 15489 to be compatible with EHR systems. Despite the amount of hospitals transitioning to EHRs, the movement is being met with some hesitance. This hesitancy could be attributed to fears that come with opting for a new system, especially as many hospital staff are used to using traditional paper methods for record keeping.

\section{Literature Review}

Many studies have been conducted to find out the barriers on EHR adoption. Gagnon et al (2016) address barriers to EHR adoption such as maintenance costs, lack of financial incentives, and suboptimal technology. Ferranti et al (2006) show that sharing EHRs among organizations is highly desirable. However, several standards are required to create compatible or interoperable data such as a common metadata, a common terminology, and common data structures. In addition, security is big concern for a remote access system. For example, while we can access medical records at home, that also means that someone can remotely steal our medical records containing sensitive information like a doctor's note or our financial information. The cloud computing paradigm offers eHealth systems the opportunity to enhance the features and functionality that they offer (Jpc Rodrigues, de la Torre, Fernández, \& López-Coronado, 2013). However, moving patients' medical information to the cloud implies several risks in terms of the security and privacy of sensitive health records. In this paper, the risks of hosting electronic health records on the servers of thirdparty cloud service providers are reviewed. To protect the confidentiality of patient information and facilitate the process, some suggestions for health care providers are made. Moreover, security issues that cloud service providers should address in their platforms are considered. Lee and Park (2008) addressed the issue of security in telemedicine system, recommending for biometric authentication to provide better security. Jain, Gyanchandani, and Khare (2017) mentioned that EHR gathering and sharing have become a big issue as it threatens patient privacy. Tseng, Yang, and Liu (2016) addressed that Taiwan's EHR exchange center (EEC) system needs a mechanism for patients to agree to disclosing their EHRs. Currently patients must agree to get full access when transferring EHRs among institutions. Tseng, Yang, and Liu (2016) developed privacy policies and patient privacy protection mechanisms. Electronic health technology has great potential to enhance the healthcare service and expand access to various health services. Wearable technology like a smart watch can host and produce medical data. However, digital-based health systems also raise various privacy and security issues. According to a recent survey (Kotz, Gunter, \& Kumar, 2016), $69 \%$ of clinicians said their organization did not address security vulnerabilities in medical devices approved by the U.S. Food and Drug Administration (FDA). According to ISO (2012), "Business Continuity is defined as the capability of the organization to continue delivery of products or services at acceptable predefined levels following a disruptive incident" (p. 24). Business continuity in medical organizations is very critical. Access to EHRs should be available for use with no or minimal disruption in terms of health system failure and unavailability of medical records. What happens if the power goes off? Where can medical professionals find out what medications their patients are on? Mansoori et al (2014) study showed how health systems provide continuous access to EHR: health systems have two servers in separate locations to back each other up. Nguyen, Bellucci and Nguyen (2014) expound on benefits and issues associated with electronic health records. One of the major findings for their study (Nguyen et al., 2014) is business continuity of EHRs. As more digital information is available online, digital divide becomes an issue. DiMaggio and Hargittai (2001) define digital divide as inequality between people haves and people have-nots access to or use of the new technologies such as health information. An example is population segments of people less likely to be online, such as the elderly and the poor. Access to EHRs for the elderly can be highly beneficial as they are able to do many things remotely instead of visiting hospitals. However, the elderly may have a hard time using various technologies. Many studies show 
negativity and distrust for electronics, widening the digital divide. Monegain (2017) found that the digital divide is a barrier to achieve population health success. According to 2017 Black Book report (2017), 70\% of Americans distrust health technology, showing a sharp increase from 10 percent in 2014. Livernois (2017) mentioned about Black Book's survey on digital divide. Black Book's survey showed that health related technologies are failing to create positive and wide use of the healthcare system as patient information technology adoption declined in 2017. Hall addressed that usability of information technology remains a concern for elders. These older patients might need help learning how to access a patient portal (Hall, 2014).

\section{Methods}

When it comes to EHR implementation, Korea is considered one of the early adopters. With the advancement of network infrastructure and support from government, most hospitals have implemented EHR systems (Fuad \& Hsu, 2012). Therefore, we can benefit from the issues and solutions of EHR systems in Korea. This study chooses to investigate the cases in Korea in order to gain valuable data on institutions which are very quickly adopting EHRs. Survey data from the five major hospitals in Korea were collected in order to identify the major issues of EHRs in Korea. An online questionnaire with open-ended and closed-ended questions was distributed to the medical staff working for hospitals in Seoul, Korea. The survey took from a sample pool of 100 hospital staffs. Out of the 100, 32 responded.

This survey was given in order to measure the overall perception and attitude about EHRs. Specifically, questions related to challenges, benefits, and favorability were asked. This provided the opportunity to allow baseline knowledge about EHR systems, ongoing implementation processes, and issues related to the transition between the two systems. Measurements taken for data analysis were chosen to identify various issues that had promoted or hindered the adoption of EHRs. Data for this study were collected during a 60-day period from the 12th of October 2016 to the12th of December 2016. As shown in Figure 1, 32 participants completed the survey. The majority of the sample populations $(88 \%)$ are Koreans, with a mean age of $43.59 \%$ of the participants claimed themselves as medical professionals.

\section{Data Analysis and Discussion}

Since the respondents all have EHRs in their organizations, the study first seeks to understand how EHRs work. The survey asked, "are you in favor of Electronic Health Records?" As shown in Figure 2, 65\% of the respondents gave answers that were in favor of EHRs. One of the respondents agreed that they were in favor "since this will be the future direction." Another one said a "great service serves people better," referring to EHRs. Some participants expressed negative comments, with one claiming, "to be honest, I'm not favor of EHRs at all because security is not guaranteed."

Survey results showed that $83.3 \%$ of respondents agreed that EHRs are an effective means to perform their daily work. This number reflects high favorability. It is also worth noting that one of the interviewees' comments was mixed with positive and negative aspects of EHRs:

Participant 1: "Medical services still can be done via direct things rather than electronic ones. Besides, the services are still the stage of the beginning. Thus, some medical accidents can happen due to awkward skills. However, it would be helpful to the elders living in country sides in Korea for they have to move to the downtown from their living place and the distance between the hospitals and their houses is really far away."

The survey also asked, "what are the benefits of Electronic

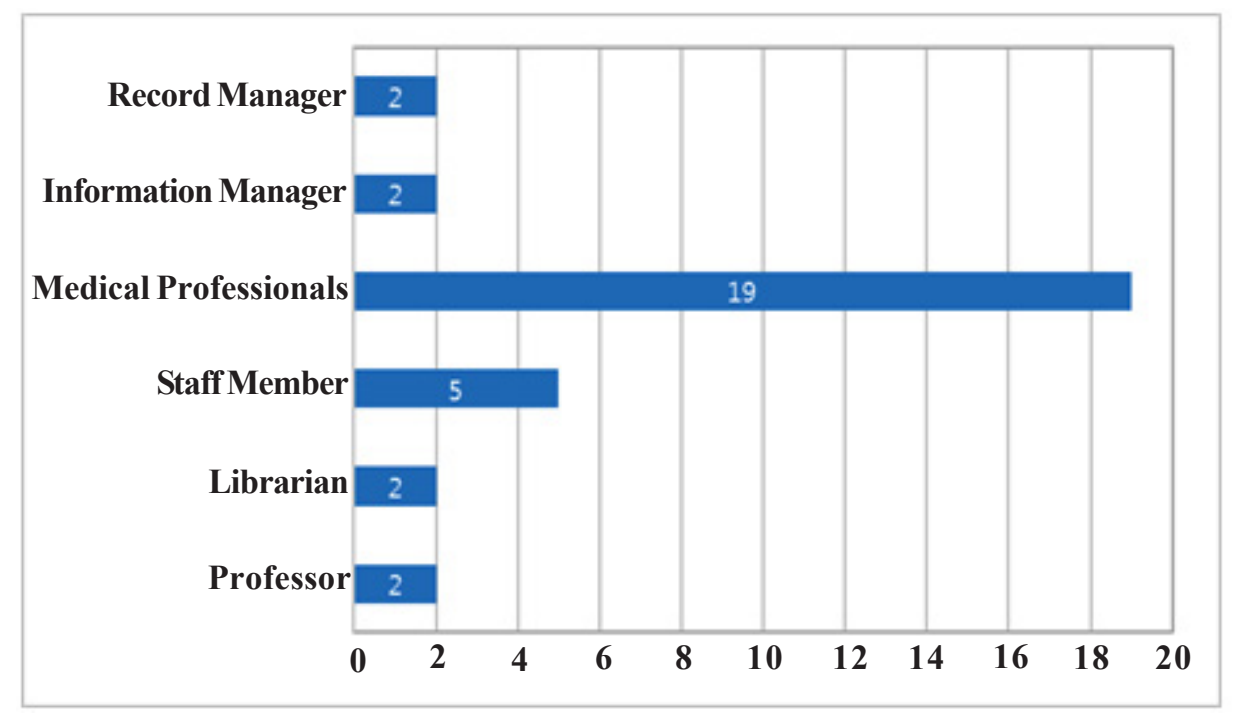

Figure 1. Job Description of Participants 


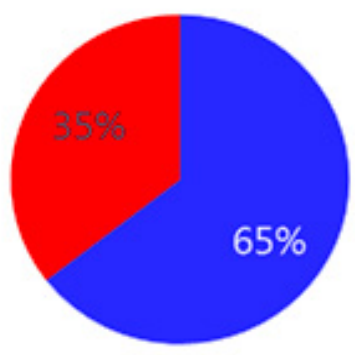

- Yes $=$ No

Figure 2. Favorability of EHRs

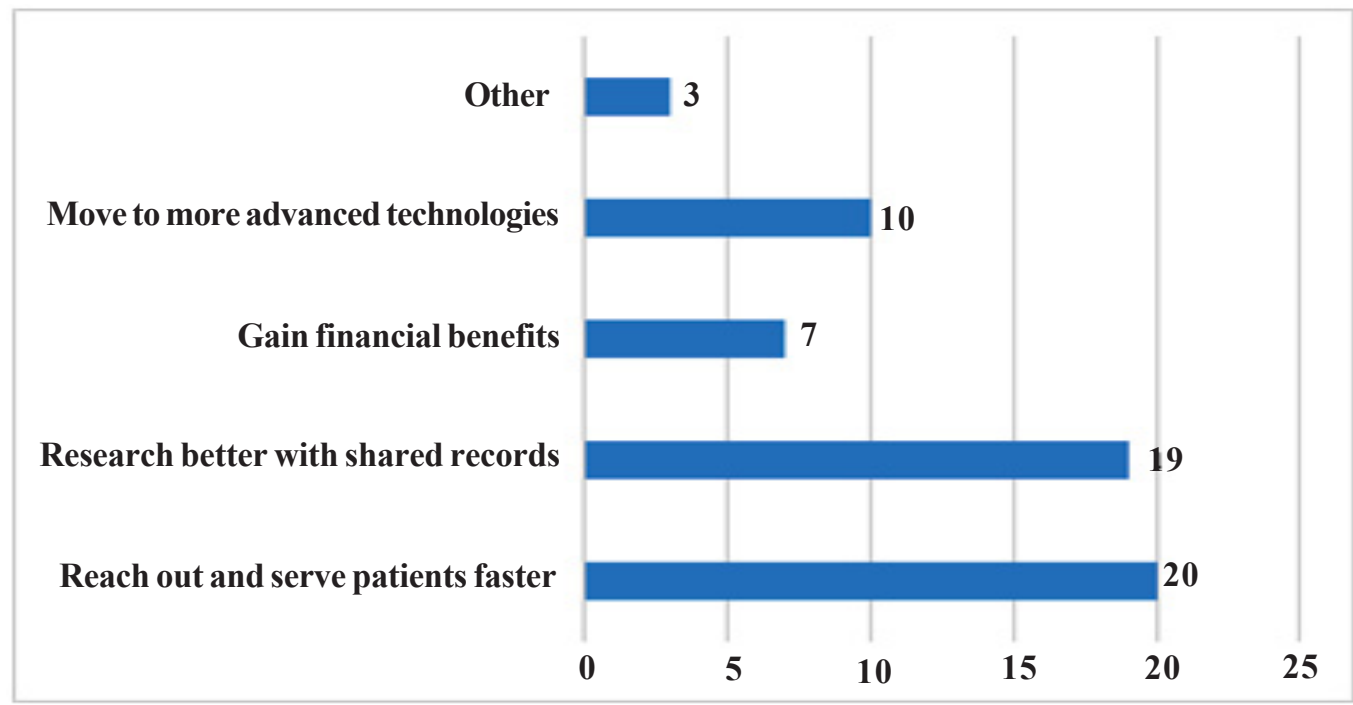

Figure 3. Benefits of EHRs

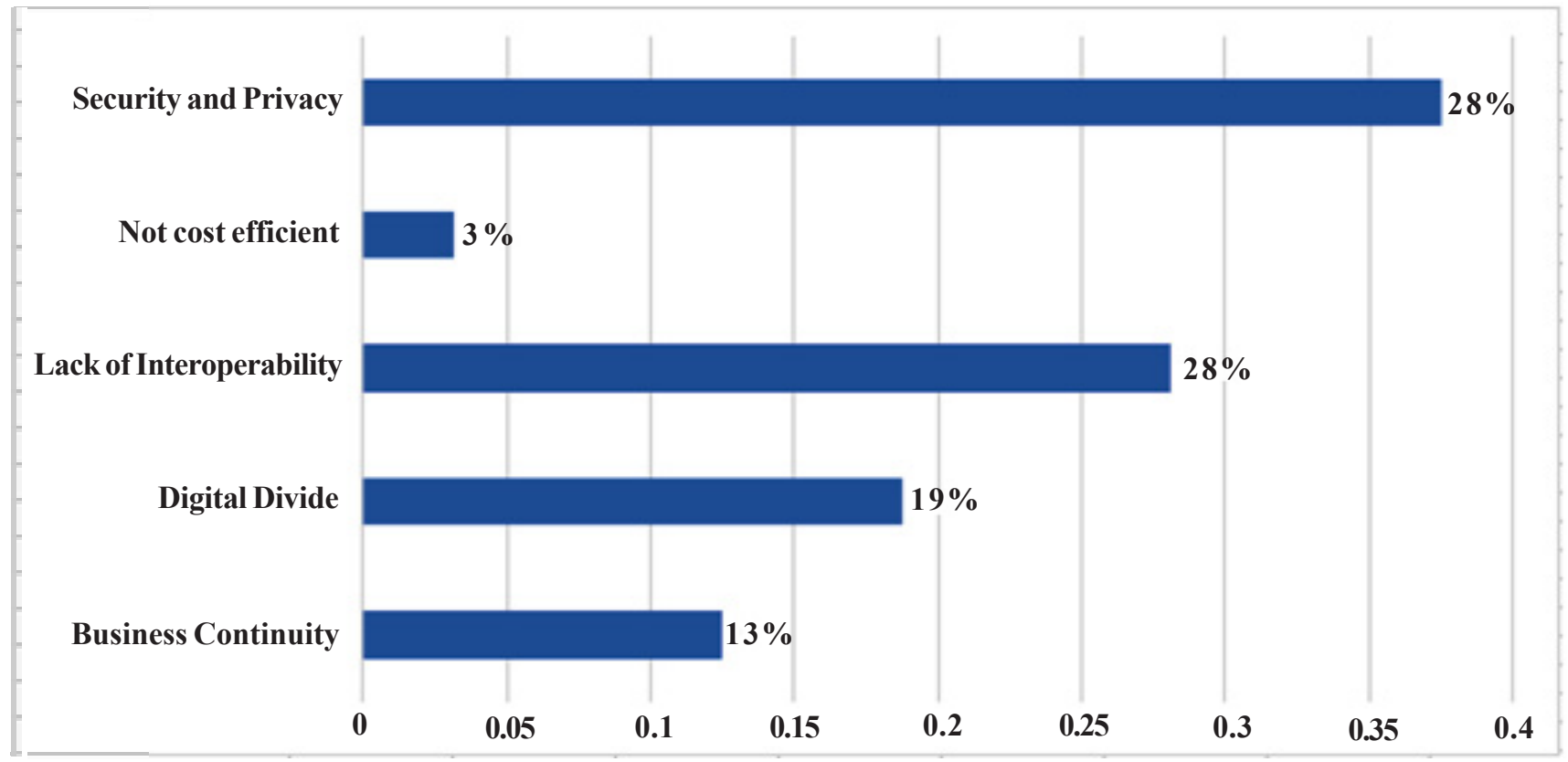

Figure 4. Issues with EHRs 
Health Records?" As shown in Figure 3, participants shared two majors benefits of EHRs. Medical organizations may do research better with shared records and reach out and serve patients faster.

\section{Major Issues of EHRs}

The survey asked, "what are the challenges of Electronic Health Records?" As shown in Figure 4, security and privacy, lack of interoperability, digital divide, and business continuity are four major issues of EHRs reported by respondents.

\section{Data Interoperability}

The use of EHRs has multiple benefits to healthcarerelated organizations. However, those advantages would be greater if interoperability of EHRs between heterogeneous health systems were achieved. When medical records are saved or retrieved, various aspects of the records are saved. Many studies (Brailer, 2005; Brino, 2014; Doumbouya, Kamsu-Foguem, Kenfack, \& Foguem, 2014; Walker et al., 2005) emphasize and show the effectiveness of searching and sharing EHRs. Lack of interoperability standards and solutions has been a major obstacle in the exchange of healthcare data between different stakeholders. To maximize the benefits of EHRs for research purposes and to serve patients, the data must be compatible with all organizations. When authorized people or organizations send and receive medical records, the records should be compatible even though they use different systems. As shown in Figure 5, the survey results reveal that interoperability issues $(28 \%)$ are one of the major concerns people have when using EHRs. In the U.S., some movements and laws are being made to push for interoperability. The government had originally planned to achieve interoperability by 2017 (Brino, 2014). However, due to these newly passed laws, the U.S. required hospitals and physicians to provide EHRs by 2015. According to Pear (2010), if they did not provide EHRs by 2015, they would be subjected to financial penalties under Medicare. In the case of EHR interoperability, several standards are under development for this purpose, such as open EHR, CEN-13606 and HL7.

Scholars address interoperability in various ways. Walker et al (2005) defined four levels for interoperability between health information systems: 1) Non-electronic data (e.g. mail, telephone), 2) Machine-transportable data (e.g. faxed or scanned documents), 3) Machine-organizable data (e.g. e-mail, proprietary file formats), and 4) Machineinterpretable data (e.g. structured data within standardized messages). Another approach to achieve interoperability is Health Level Seven (HL7). HL7 is an approved protocol for managing the exchange of information related to medical data (HL7 Internantional, 2017). HL7 has identified functions to share health records and is promoted by many organizations. Hwang (2010)designed the HL7 interface engine to exchange medical records among different clients and servers. Cho and Kwon (2008) also proposed
$\mathrm{HL} 7$ as a standardized protocol to share and exchange medical information among medical clinics. Adebesin et al (2013) also provide four levels of interoperability. The first level is the technical level that permits heterogeneous systems to exchange data, but does not ensure that the receiving system will be able to use the exchanged data in a meaningful manner. The second level is the syntactic level that ensures the preservation of the clinical purpose during transmission among healthcare systems. Third is the semantic level that permits multiple systems to interpret the information that has been exchanged in a similar manner through pre-defined shared meaning of concepts. Fourth is the organizational level. This level makes it easy to integrate business processes and workflows beyond the limits of a single organization. One of the major interoperability issues is at the semantic level. Exchanging compatible file formats and other technical level interoperability issues can be resolved with agreement among stakeholders. However, semantic interoperability is hard to achieve because a word has multiple meanings and relationships among the words are not explicit. According to Kwasnik (2004), words have meaning and the meaning has several layers: 1) Lexical level, 2) Semantic level, 3) Syntactic level, 4) Discourse level, and 5) Pragmatic level. Lexical level of a word means simply the word itself without grammar or meaning. Semantic level represents a meaning of a word. In syntactic level, the relationship of the words to each other are shown, known as grammar. In discourse level, the meanings of a word are interpreted in the context of text that is greater than the single sentence. In pragmatic level, the meaning of a word is embedded in world knowledge, that is, the way they are used. When doctors write notes as records, meaning of the records may be different by context or by culture.

Another issue is relationship between words. When we share medical records among different organizations or different languages, we face many problems. For example, patients use "cancer" and medical professionals use "neoplasm" to refer to the same thing. When hospitals and doctors implement EHRs, they should pay attention to interoperability issues using international standards. At the semantic level, ontology must be in place before systems are implemented. Without appropriate ontology, data is not interoperable nor shareable among hospitals or organizations. Medical ontology means shared vocabulary within a medical community.

Medical ontology can be a great solution for semantic interoperability because it provides a domain of words and a set of relationships of words to EHR systems. For example, cancer and neoplasms are synonyms and they are in the "disease" domain. As shown in Figure 5, OWL (Web Ontology Language) can show an equivalent relationship between "cancer" and "neoplasm" in the disease domain.

The example demonstrates how an EHR system was able to process two different words, despite the fact that those 


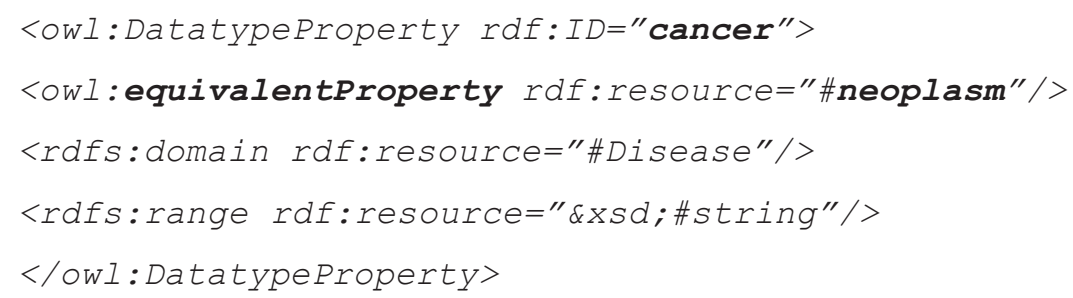

Figure 5. Relationship between Cancer and Neoplasm in OWL

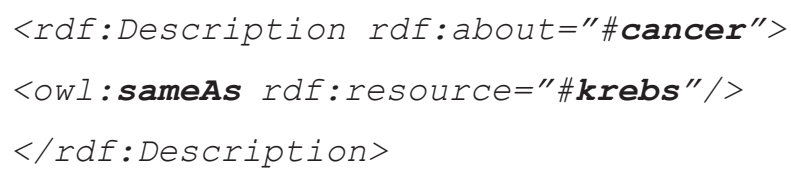

Figure 6. Cross-language in OWL

words are used different terms. This semantic interoperability was achieved by using an ontology. In addition to connecting synonyms, an ontology may also be used as a cross-language EHR system. As shown in Figure 6, "cancer" in English and "krebs" in German are shown and an EHR system can then understand the words in different languages.

In order to exchange and share EHR data, semantic interoperability must be guaranteed. Often times hetergeneous EHR systems are implemented at hospitals; the result is that these hospitals are unable to fully share and exchange their EHR data and understand the meaning and relations of the records. However, an ontology can resolve these semantic interoperability issues.

\section{Security}

Anderson (2010) defined information security as such: a "well-informed sense of assurance that the information risks and controls are in balance"(p. 42). EHR systems may have many security vulnerabilities and one of the threats for EHR systems is malicious code. Weaknesses in software and hardware must be removed to minimize vulnerability, so that risk and controls are balanced to protect the system and to continue business. It is dangerous for EHRs to have security issues since medical records usually require patients to fill out sensitive and accurate personal information and for doctor's to record confidential details, like doctor's notes, lab diagnoses, prescription information, and personal and insurance related information. Hospitals and doctors are especially concerned about doctor notes in EHRs. While the patient chart and laboratory results are important, the information containing the doctor's opinion and laboratory results is also very important due to their role in diagnosing. This nature of medical records makes them a main target for hackers. Stolen medical records may be used to commit identity theft. Humer and Finkle's (2014) study shows that medical records have the most number of incidents when it comes to security breach in hospitals. According to Identity Theft Resource Center (ITRC), the number of medical identity thefts are increasing and the number of medical identity thefts exceeds the number of financial identity thefts (Rocha, 2013). A survey last year by the Ponemon Institute estimated the cost of Medical Identity Theft to consumers to be $\$ 12$ billion for 2013 (Munro, 2014). The larger consumer risk is not financial - it's the lifethreatening inaccuracies in the medical records themselves (often used for committing the financial fraud). Therefore, proper and secure management of medical records is essential. With the possibility of losing medical records, individuals and organizations are reluctant to use EHR systems. As shown in Figure 7, the survey results reveal that security and privacy issues (38\%) are one of the major concerns people have when using EHRs. One of the survey questions also measured participants' favorability on EHRs. Most participants who do not favor EHRs answered privacy of personal information as one of the major reason for their negative answer.

Whilst traditional security is more simple - everyone knows how to lock a cabinet - security in the digital age is very complicated. In order to achieve the three security goals of Confidentiality, Integrity, and Availability, three security controls must be in place. These security controls include managerial, physical, and technical security (ISC2, 2010). For managerial security, security policies can guide how technical security controls can be implemented into a hospital's network. Security policies also provide access control list for doctors, patients, and staff members. Each individual should have different access levels to EHRs since their roles and responsibilities are different. For physical security, CCTV and security staffs are some controls to protect physical assets. Technical security includes firewalls, Intrusion Detection System (IDS), Public Key Infrastructure (PKI), and Single Sign On (SSO).

The goal of confidentiality is to protect EHRs. With cryptographic algorithms, EHRs are encrypted. To see 


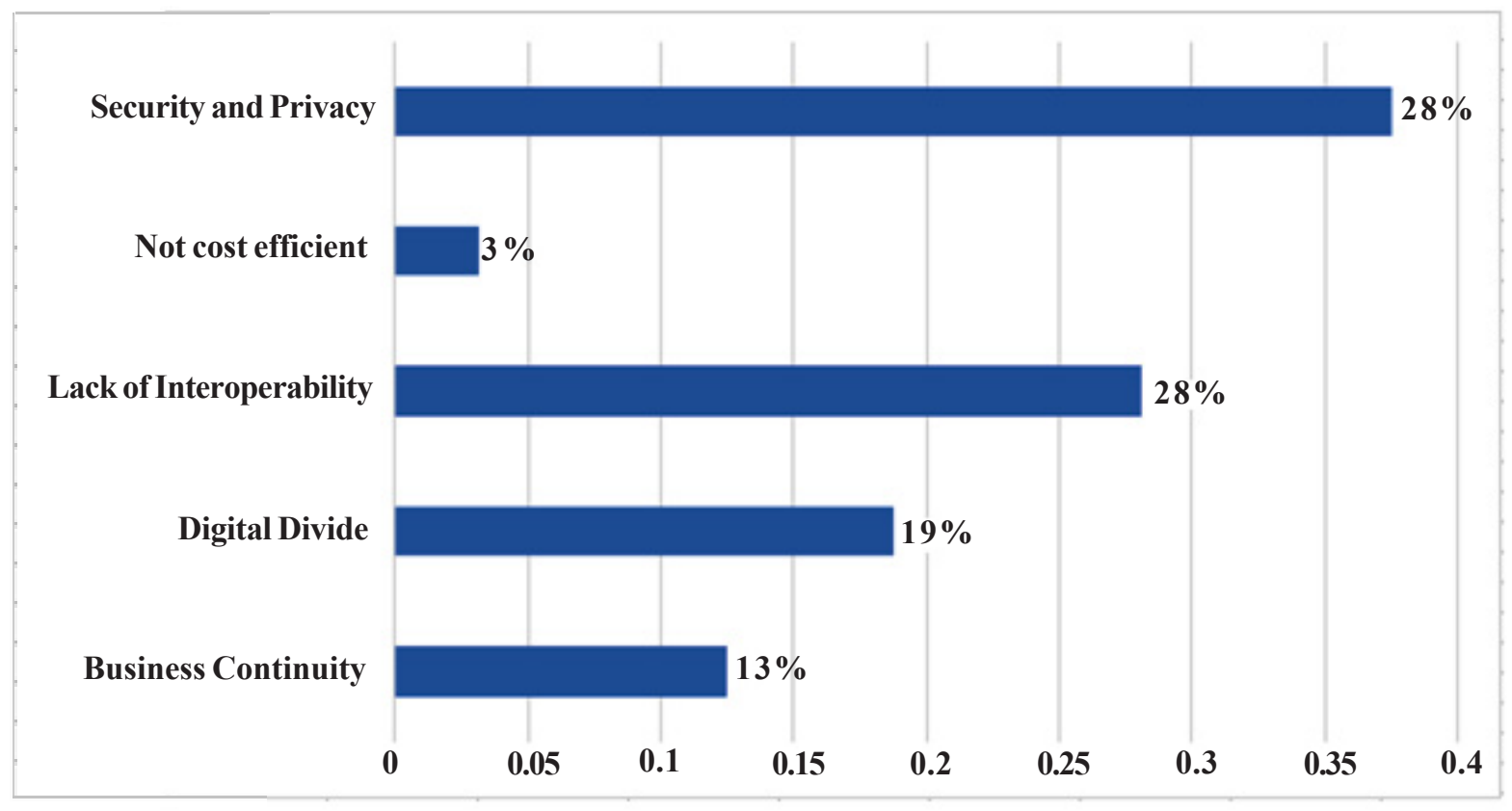

Figure 7. Issues with EHRs

the encrypted data, a key would be needed to decrypt the data. The records should be encrypted in case unauthorized users try to obtain the medical records. Using weak cryptographic algorithms such as IDEA or DES is not a good strategy to provide confidentiality. The goal of integrity is to maintain the trustworthiness of EHRs. Integrity of medical records is very crucial because medical records contain very critical information such as lab results and treatment. Since we can easily change electronic records without leaving any traces, managerial and technical security controls must be provided to ensure integrity. Segregation of duties and logins must be in place to help provide and uphold the integrity of medical records. Segregation of duties assures roles and responsibilities of medical professionals and staff members are clearly outlined and performed. The goal of availability is to make EHR accessible. Availability in security refers to the provision of resources whenever resources are demanded. The availability of medical records is critical. When a doctor performs medical practice, medical records should be available at all times. For example, the medical records or vital signs should be displayed while the doctor performs surgery in the operation room. The patients' records should be available all the time as well. When a hospital transfers patient records to other hospitals, the availability of EHR systems are critical. Medical professionals use various devices such as smartphones or tablet computers to send and receive EHRs, which must be available no matter what devices users use. Redundant network devices such as routers or switches can increase availability of EHRs. Cold, warm, and hot sites are examples of backup sites in case a natural disaster happens at the main site where EHRs are stored. Protection from network attacks such as distributed denial of service (DDOS) also assures availability of EHRs.
To conclude, security controls are used to achieve three security goals of confidentiality, integrity, and availability. For example, we can use technical security controls such as virus detection software packages to protect EHR systems, and ensure availability without interruption. Another example deals with internal employees' accidental unauthorized access, which is how most security incidents occur. With a lack of computer security skills by the hospital staff, accidental security breaches become more susceptible and probable. To avoid such accidental and unauthorized access, security awareness and training should be established.

\section{Business Continuity}

Business continuity is the ability of an organization to maintain essential functions during and after a disaster has occurred. Business continuity planning (BCP) is a methodology used to create a plan for maintaining continuous business operations before, during, and after disaster events (Snedaker, 2013). Network failure, data loss, hardware failure, software failure, and attacks are some examples of disruptions on EHR availability. For the business to properly function, EHRs must always be available over the network. To ensure network availability, BCP must be in place. BCP ensures that a hospital can continue to operate in case of security incidents and is able to restore its business to an operational state. With the possibility of network, software, and hardware failures, hospitals are reluctant to use EHR systems. Using electronic format of health records can be scary without immediate technical support by IT staff in house. Digital data are prone to damage by malicious code and hospitals may lose access to EHRs. In the survey, business continuity was highlighted as a major concern by hospital staff members working in Korea. Participants expressed 
their concerns on the unstable and unreliable business continuity that may come as a risk of implementing EHRs. In fact, $45 \%(\mathrm{~N}=27)$ of the respondents were concerned about business continuity on EHRs. Even though BCP is a major concern for medical professionals; solutions are available to resolve business continuity issue. Business continuity planning must be in place to expand EHRs. Without assurance of business continuity, physicians are reluctant to adopt EHRs. When disruptions occur, business continuity planning will allow the hospital to immediately resume operations. Business continuity planning should include roles and responsibilities of EHR services. For example, when viruses affect some computers, there should be a specialist in-charge that can take care of the problem. In order to minimize the risk such as data loss, network failures, virus issues, hardware, software issues, and accidental staff access, hospitals need a BCP. A practical approach to BCP is using a cloud-based EHR rather than an in-house system. Cloud-based EHR systems can deliver greater security than paper records (McKee, 2014). Paper records can be lost in natural disasters or fires but cloud-based records are backed up in several locations to protect them (McKee, 2014). Polack (2011) compares the pros and cons of cloudbased EHR systems. According to Polack (2011), it is cheaper to set up the system of cloud-based EHRs, and it is convenient for physicians who are not office-based. It is beneficial for small offices as well. However, cloud service providers must ensure that security mechanisms, backup data, and BCP are in place to provide business continuity.
Users of cloud-based EHR must be kept informed about how their data are being managed.

\section{Digital Divide}

Digital divide is the term used to define the gap between people who have and people who do not have access to technology, as well as people who know how to use technology and people who do not know how to use technology; it is the difference between the technological "haves" and "have nots" and "know" and "do not know." Digital divide will leave many patients behind and they will not be able to get the full benefits or services of EHRs. Some studies (DiMaggio \& Hargittai, 2001; Tierney et al., 2002) address digital divide issues in developing countries. Tierney et al (2002) mentioned that basic technologies to promote EHRs are often not available in developing countries. Maisie et al (2004) developed a personal health information management system (PHIMS) to address the digital divide issue. Regardless of the availability of network infrastructure, some people are not familiar with computers and the Internet. There are some people who do not have computers or skills to use EHRs. It is hard enough even if you do have a computer and skills. If you do not have either or one of those things, this may result in an even greater digital divide for EHR use. One of the survey questions measured the importance of digital divide for EHRs. As shown in Figure 8, results of our survey show concerns about the digital divide in EHRs.

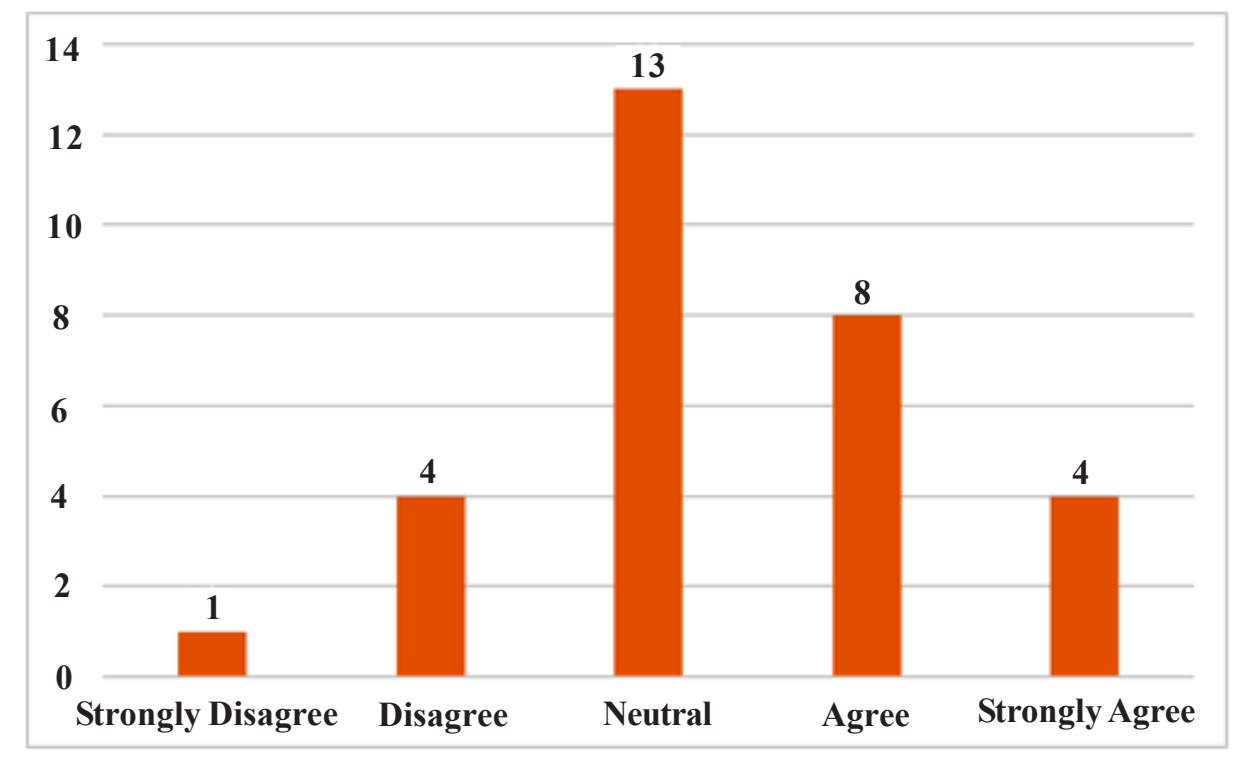

Figure 8. Digital Divide

\begin{tabular}{|l|l|l|}
\hline Category & User & Provider \\
\hline Lack of Technology Access & People with restricted access & EHRs related devices \\
\hline Lack of Technology Literacy & Information literacy & Lack of training \\
\hline
\end{tabular}

Table 1. Types of Digital Divide 
Many respondents showed their concern about digital divide from both the side of the provider and the user. From the provider's side, they were concerned about the difficulty of integrating with in-house IT and the difficulty of learning new technology and not having enough ability to implement EHRs. From the user's side, they were concerned about the difficulty of learning new technologies.

As shown in Table 1, there are four types of digital divide. From a user's perspective, either people in remote or rural areas, for example, have limited access to technology or people do not know how to use technology. From a provider's perspective, either provider do not have EHRs related equipment or do not know how to use EHRs related software.

To minimize the digital divide on lack of technology literacy, international agencies and corporations should help developing countries in the areas of technology and various resources to enhance healthcare services (Tierney et al., 2002). Hospitals must provide computer literacy programs to patients. Without sufficient training on the use of computers and other devices for EHRs, digital divide will become a major barrier for EHR implementation. The digital divide in medical terms is also an issue because people may have a difficult time understanding medical terms. This can lead to confusion to understand doctor's notes and description of prescriptions which has a negative impact on a patient and treatment plan. Boden (2009) proposes the use of consumer health vocabularies (CHV) and natural language processing (NLP) in order to improve people's understanding of medical professionals' terms. As shown in Figure 6, medical ontology written in OWL can be used to minimize gaps between doctors and patients. To enhance the technology access and information literacy of users, the Korean government has utilized the "Information Network Village" to increase it. Information Network Village is a facility to provide computer training in rural areas (Kang, 2002). After the Korean government adopted the information network village in 2001, computer availability in rural areas rose from $29 \%$ to $72 \%$ and Internet usage increased from $11 \%$ to $66 \%$ (Choo, 2001).

\section{Conclusion}

Compared to paper medical records, EHRs allow for the efficient sharing of medical records with other organizations. A high EHR participation rate of medical institutions will lead towards efficient collaboration among said institutions. On the other hand, a low participation rate will render useless the benefits of EHRs. If medical institutions implement EHRs effectively, medical institutions can then offer faster patient service, an increase in mobility of medical records, convenience for its citizens, and seamless medical research collaboration. Even though EHRs have many advantages, stakeholders are hesitant, and at times even fearful of adopting EHRs into their medical systems, due to the many dangers and risks that plague EHRs.
This study identifies four major issues in the use of EHRs and provides solutions to each of these issues in order to promote effective adoption of EHRs. In order to properly implement EHRs, medical organizations and professionals need to be aware of the four main issues of implementation: interoperability, security, business continuity, and digital divide. These issues can be mitigated by promoting awareness of these obstacles and addressing them alongside the implementation process. Therefore, medical institutions should apply the recommended solutions this paper offers in order to safeguard against the potential risks and threats of these issues. There exists means to properly implement EHRs which effectively negates possible drawbacks. Interoperability issues can be avoided by considering metadata as a standard to create compatible health information systems. For security issues, medical institutions can minimize accidental and unauthorized access with security awareness training. Accessing files from a cloud-based EHR resolves business continuity issues. Finally, issues with the digital divide can be avoided by creating training programs for patients which focus on operating the various devices and services of EHRs.

Participation pool of this paper's primary research was limited to hospitals in South Korea. Because of this, the findings of this paper may not be fully applicable to other cities and countries. This paper recommends further studies to conduct research in other cities or other countries. To extend the study to a global level will contribute to the body of knowledge in EHRs. More research to promote adoption of EHRs will allow us to collaborate between countries to resolve rare diseases and perform research on medicine. Indeed, EHRs can benefit from a more globalized community; the more countries that adopt EHRs, the more information can be distributed internationally. The result will allow the ease of international medical collaboration which can lead to a more effective global medical community. As security and business continuity are most concerning for users, further studies may be conducted on the issues on EHRs. There is no such thing as $100 \%$ security when using EHRs. Even though many security systems are installed, medical systems are still vulnerable to numerous types of threats. Research to protect EHRs should be continued in order to achieve a higher level of security, ensure integrity, make EHRs available and updated, and sustain business continuity.

\section{References}

[1] Adebesin, F., Foster, R., KotzT, P., Greunen, D. V. (2013). A review of interoperability standards in e-Health and imperatives for their adoption in Africa. South African Computer Journal, 50(1), 55-72.

[2] Anderson, R. J. (2010). Security Engineering: A Guide to Building Dependable Distributed Systems. New Jersey: John Wiley \& Sons.

[3] Black Book Research. (2017). Healthcare's Digital 
Divide Widens, Black Book Consumer Survey. Retrieved from https://blackbookmarketresearch.newswire.com/ news/healthcares-digital-divide-widens-black-bookconsumer-survey-18432252

[4] Boden, C. (2009). Overcoming the linguistic divide: a barrier to consumer health information. Journal of the Canadian Health Libraries Association, 30(3), 75-80.

[5] Brailer, D. J. (2005). Interoperability: The Key To The Future Health Care System. Health Affairs, 24, W5-19W15-21.

[6] Brino, A. (2014). Senators press for EHR interoperability. Retrieved from http://www.healthcareit news.com/news/senators-press-ehr-interoperability

[7] Carpathia. (2013). 5 Benefits of EMR vs. Paper Medical Records. Retrieved from http://carpathia.com/blog/5benefits-of-emr-vs-paper-medical-records/

[8] Chae, Y. M., Yoo, K. B., Kim, E. S. (2010). The Adoption of Electronic Medical Records and Decision Support Systems in Korea. Health Informatics Research, 17(3), 172-177.

[9] Cho, I., Kwon, H. (2008). Design and Implementation of Electronic Medical Record System Based on HL7-CDA for the Exchange of Clinical Information. The Korean Institute of Communications and Information Sciences 33(5), 379-385.

[10] Choo, S. (2001). The Use of Networked Computer and Changes of Life in the Rural Area : The Case of an Information Model Village. Journal of Korea Planning Association, 36(6), 137-151.

[11] DiMaggio, P., Hargittai, E. (2001). From the 'digital divide'to 'digital inequality': Studying Internet use as penetration increases. Princeton: Center for Arts and Cultural Policy Studies, Woodrow Wilson School, Princeton University, 4(1), 4-2.

[12] Doumbouya, M. B., Kamsu-Foguem, B., Kenfack, H., \& Foguem, C. (2014). Telemedicine using mobile telecommunication: Towards syntactic interoperability in teleexpertise. Telematics and Informatics, 31(4), 648-659. doi:http://dx.doi.org/10.1016/j.tele.2014.01.003

[13] Ferranti, J. M., Musser, R. C., Kawamoto, K., Hammond, W. E. (2006). The Clinical Document Architecture and the Continuity of Care RecordA Critical Analysis. Journal of the American Medical Informatics Association, 13(3), 245-252. doi:10.1197/jamia.M1963

[14] Fuad, A., Hsu, C. (2012). High rate EHR adoption in Korea and health IT rise in Asia. International Journal of Medical Informatics, 81(9).

[15] Gagnon, M. -P., Simonyan, D., Ghandour, E. K., Godin, G., Labrecque, M., Ouimet, M., Rousseau, M. (2016). Factors influencing electronic health record adoption by physicians: A multilevel analysis. International Journal of Information Management, 36(3), 258-270.

[16] Gunter, T., Terry, N. (2005). The Emergence of National Electronic Health Record Architectures in the United
States and Australia: Models, Costs, and Questions. Journal of Medical Internet Research, 7(1).

[17] Hall, S. D. (2014). Are EHRs creating a digital divide for the elderly? Retrieved from http://www.fiercehealthcare. com/ehr/are-ehrs-creating-a-digital-divide-for-elderly

[18] Hersh, W. R., Weiner, M. G., Embi, P. J., Logan, J. R., Payne, P. R. O., Bernstam, E. V., Saltz, J. H. (2013). Caveats for the Use of Operational Electronic Health Record Data in Comparative Effectiveness Research. Medical care, 51(8 0 3), S30-S37. doi:10.1097/ MLR.0b013e31829b1dbd

[19] HL7 Internantional. (2017). Introduction to HL7 Standards. Retrieved from http://www.hl7.org/implement/ standards/index.cfm?ref=nav

[20] Hsiao, C.-J., Hing, E., Socey, T. C., Cai, B. (2011). Electronic Health Record Systems and Intent to Apply for Meaningful Use Incentives Among Office-based Physician Practices: United States, 2001-2011. Retrieved from http://www.cdc.gov/nchs/data/databriefs/db79.htm

[21] Humer, C., Finkle, J. (2014). Your medical record is worth more to hackers than your credit card. Retrieved from http://www.reuters.com/article/us-cybersecurityhospitals-idUSKCNOHJ21/20140924

[22] Hwang, D. (2010). Implementation of HL7 Interface Engine for Medical Information Exchange. Journal of the Korea society of computer and information, 15(8), 89-98.

[23] ISC2. (2010). 10 (ISC)² CISSP domains. Retrieved from https://www.isc2.org/cissp/default.aspx

[24] ISO. (2012). ISO 22301 Business Continuity Management. Retrieved from https://www.bsigroup.com/ en-GB/iso-22301-business-continuity/

[25] Jain, P., Gyanchandani, M., \& Khare, N. (2017). Privacy and Security Concerns in Healthcare Big Data: An Innovative Prescriptive. Journal of Information Assurance \& Security, 12(1), 18-3

[26] Jha , A. K., DesRoches , C. M., Campbell , E. G., Donelan , K., Rao , S. R., Ferris , T. G., Blumenthal, D. (2009). Use of Electronic Health Records in U.S. Hospitals. New England Journal of Medicine, 360(16), 1628-1638. doi:10.1056/NEJMsa0900592

[27] Jpc Rodrigues, J., de la Torre, I., Fernández, G., López-Coronado, M. (2013). Analysis of the Security and Privacy Requirements of Cloud-Based Electronic Health Records Systems. Journal of Medical Internet Research, 15(8), e186. doi:10.2196/jmir.2494

[28] Kang, J. (2002). An Introduction to the Policies of Information Network Village.

[29] Kotz, D., Gunter, C. A., Kumar, S. (2016). Privacy and Security in Mobile Health: A Research Agenda. Computer, 49(6), 22-30.

[30] Kwasnik, B. (2004). Revisiting the Preserved Context Index System (PRECIS): The Bridge between Hierarchically Structured Thesauri and Facetted 
Classifications. Paper presented at the ASIST SIG/CR Workshop.

[31] Lacktman, N. (2014). Telehealth Opportunities Arise as South Korea Opens Door to Telemedicine. Health Information Technology. Retrieved from http:// www.healthcarelawtoday.com/2014/04/02/telehealthopportunities-arise-as-south-korea-opens-door-totelemedicine/

[32] Lee, A., Kang, D., Baik, D. (2009). A Design of Metadata Based EMR System for Efficient Data Use. Paper presented at the Korea Information Science Fall Conference, Seoul, Korea.

[33] Lee, E.-M., Kim, M., Yim, J. H. (2012). A Study on the Current Status and Tasks of Medical Records Management : Focused on Applying the KS X ISO 15489 to the $Y$ Hospital. [A Study on the Current Status and Tasks of Medical Records Management : Focused on Applying the KS X ISO 15489 to the Y Hospital]. Journal of the Korean Society for Information Management, 29(3), 257-285.

[34] Lee, H. (2013). EMR preservation using authoratative third party. Hanyang Law Review, 24(4), 123-149.

[35] Lee, Y., Park, D. (2008). A Telemedicine System Based on Device Authentication in the Wire and Wireless Environments. [A Telemedicine System Based on Device Authentication in the Wire and Wireless Environments]. The Journal of The Korean Institute of Communication Sciences, 33(10), 359-364.

[36] Livernois, C. (2017). 5 reasons why healthcare's digital divide is growing. Retrieved from http://www.clinicalinnovation.com/topics/analytics-quality/top-5-causeshealthcare-s-digital-divide

[37] Maisie, W., Lau, C., Matsen, F. A., Kim, Y. (2004). Personal health information management system and its application in referral management. IEEE Transactions on Information Technology in Biomedicine, 8(3), 287-297. doi:10.1109/TITB.2004.834397

[38] Mansoori, B., Rosipko, B., Erhard, K. K., Sunshine, J. L. (2014). Design and Implementation of Disaster Recovery and Business Continuity Solution for Radiology PACS. Journal of Digital Imaging, 27(1), 19-25. doi:10.1007/s10278-013-9625-4

[39] McKee, S. (2014). 5 Advantages of a Cloud-Based EHR for Small Practices. Retrieved from http:// www.poweryourpractice.com/practice-management/5advantages-of-a-cloud-based-ehr-for-small-practices/

[40] Monegain, B. (2017). Healthcare's digital divide is getting bigger and other bad news from Black Book.
Retrieved from http://www.healthcareitnews.com/news/ healthcares-digital-divide-getting-bigger-and-other-badnews-black-book

[41] Munro, D. (2014). New Cyberthreat Report By SANS Institute Delivers Chilling Warning To Healthcare Industry. Retrieved from http://www.forbes.com/sites/danmunro/ 2014/02/20/new-cyberthreat-report-by-sans-institutedelivers-chilling-warning-to-healthcare-industry/

[42] Nguyen, L., Bellucci, E., Nguyen, L. T. (2014). Electronic health records implementation: An evaluation of information system impact and contingency factors. International Journal of Medical Informatics, 83(11), 779796. doi:http://dx.doi.org/10.1016/j.jimedinf.2014.06.011

[43] Pear, R. (2010). Standards Issued for Electronic Health Records. The New York Times. Retrieved from http:// www.nytimes.com/2010/07/14/health/policy/ 14health.html? r $r=0$

[44] Polack, P. (2011). Pros and cons of cloud based or web based EMR systems. Retrieved from http:// www.kevinmd.com/blog/2011/05/pros-cons-cloud-basedweb-based-emr-systems.html

[45] Rocha, K. (2013). Medical identity theft. Retrieved from http://www.idtheftcenter.org/Medical-ID-Theft/medicalidentity-theft.html

[46] Shin, I., Chung, M. (2007). Design and Implementation of an Electronic Medical Record System based on RFID.

[47] Snedaker, S. (2013). Business Continuity and Disaster Recovery Planning for IT professionals (2nd ed.). Boston: Newnes.

[48] Tierney, W., Rotich, J., Smith, F., Nii, J., Einterz, R., Hannan, T. (2002). Crossing the 'digital divide': implementing an electronic medical record system in a rural Kenyan health center to support clinical and research. Paper presented at the AMIA Annual Symposium 2002, San Antonio, TX.

[49] Tseng, T.-W., Yang, C.-Y., Liu, C.-T. (2016). Designing Privacy Information Protection of Electronic Medical Records. Paper presented at the 2016 International Conference on Computational Science and Computational Intelligence (CSCl), Las Vegas, NV, USA.

[50] Walker, J., Pan, E., Johnston, D., Adler-Milstein, J., Bates, D. W., Middleton, B. (2005). The value of health care information exchange and interoperability. Health Aff, 5-18.

[51] Williams, F., \& Boren, S. A. (2008). The role of the electronic medical record (EMR) in care delivery development in developing countries: a systematic review. Informatics in Primary Care, 16(2), 139-145. 